\title{
Acute appendicitis revealing salpingiosis
}

\author{
Adriana Handra-Luca
}

Accepted: 8 January 2015 / Published online: 25 January 2015

(C) Springer-Verlag Berlin Heidelberg 2015

\section{Dear Editor:}

We have read with great interest the recent article on histopathological findings in 24,697 cases [1]. Microscopic examination revealed in $7.38 \%$ of the patients unsuspected findings among which endometriosis. Less frequently, remnants of salpingiosis type may also be identified in the appendix, three such cases being reported, to our knowledge, to date [2-4]. We have recently seen an additional case of salpingiosis of the vermiform appendix diagnosed incidentally in a previously unreported context to our knowledge, that of acute appendicitis.

The patient (49-year-old woman) presented with nausea, abdominal pain (elective right iliac fossa), frisson and vomiting. The patient's history revealed arthrosis. CT scan showed a retrocecal appendix with a thickened wall, "infiltrated" fat and peritoneal thickening. The diagnosis was that of retrocecal acute appendicitis, and appendectomy was performed. The appendix $(7.2 \mathrm{~cm}$ in length, analysed entirely on microscopy) showed acute panappendicitis with peritonitis. Two foci of less than $1 \mathrm{~mm}$ in size (with a distance of $2 \mathrm{~mm}$ between) composed of few subserosal epithelial glands (without interspersed stroma or endometrial-type chorion) showing peg and ciliated cells were identified, suggestive of salpingiosis. The glandular cells expressed cytokeratin 7 , and did not express cytokeratin 20 and calretinin. These glands were not seen on CD31 stained section.

Appendiceal salpingiosis is very rare. In the three cases reported to date, to our knowledge, the lesions, although multicystic and ranging $0.5-0.8 \mathrm{~cm}$, were an incidental finding on appendectomy specimen resected during surgery for

A. Handra-Luca $(\bowtie)$

APHP Service d'Anatomie pathologique, Universite Paris Nord

Sorbonne Cite, GHU Avicenne: 125 rue Stalingrad,

93009 Bobigny, France

e-mail: adriana.handra-luca@avc.aphp.fr

\author{
A. Handra-Luca \\ e-mail: adriana.handra-luca@hotmail.com
}

salpingitis, sigmoid stricture or diverticulosis and, devoid of inflammation [2-4]. The presence of disparate glandular structures in the appendix subserosa, at distance of the most intense appendicitis lesions, may suggest well-differentiated adenocarcinomatous glands, primitive or secondary, which are observed more frequently [1]. The lack of cell atypia and of tumoral stroma allowed to eliminate this possibility. Due to the minute size of the lesions seen in the present case, the CD31 immunohistochemical staining was non-contributive, the glandular structures being absent on this slide. The histogenesis of appendiceal salpingiosis is still matter of debate. A metaplastic origin cannot be completely ruled out although the lack of calretinin positivity. However, the hypothesis of mullerian remnant structures of tubal type remains the most plausible.

In conclusion, we report here a rare case of appendiceal microscopic salpingiosis diagnosed incidentally in an appendix showing acute panappendicitis. The identification of such lesions is important to be differentiated from adenocarcinomatous glands.

Acknowledgments The author thanks Dr. E Dragoescu, Pr. J Rutgers, Pr. W Zheng, Dr. L Larnaudie, Dr. V Vasiliu, the NCA/Avicenne, BIUM and CMDP/APHP teams.

\section{References}

1. Charfi S, Sellami A, Affes A, Yaïch K, Mzali R, Boudawara TS (2014) Histopathological findings in appendectomy specimens: a study of 24, 697 cases. Int J Color Dis 29(8):1009-1012

2. Cajigas A, Axiotis CA (1990) Endosalpingiosis of the vermiform appendix. Int J Gynecol Pathol 9(3):291-295

3. McCluggage WG, Clements WD (2001) Endosalpingiosis of the colon and appendix. Histopathology 39(6):645-646

4. Pollheimer MJ, Leibl S, Pollheimer VS, Ratschek M, Langner C (2007) Cystic endosalpingiosis of the appendix. Virchows Arch 450(2):239-241 